\title{
Short Story: Covid-19; A Hidden Enemy to Heart?
}

\author{
Sana e Mustafa,* Rida Fatima, Shagufta Rasheed
}

University of Agriculture, Faisalabad, Pakistan

\begin{abstract}
Most of the patients with COVID experienced heart problems, but the causes are unclear. It was predicted that pre-existing heart disorders, and oxygen deficiency might be the possible cause. But the proof that the virus can directly target the individual cells of the heart has been limited, until now.
\end{abstract}

Keywords: COVID, Oxygen

\section{Introduction}

Heart damage is most often caused by Heart attacks are mostly caused by heart damage that is due to the formation of a blood clot in a vulnerable heart artery. It ultimately leads to the blocking delivery of oxygen to the heart muscle. COVID-19-related infection increases the risk of heart attack by stimulating the body's clotting system and disturbing the blood vessel lining.

Severe Acquired Respiratory Syndrome-CoV-2, the virus that causes COVID-19, mostly affects the lungs but and also lead to serious heart problems.SARS-COV-2 directly harm the heart muscles by attacking on the straight path of balance between oxygen's supply and demand. In a study of hospitalized patients, one quarter was suffering from covid-19. They showed elevated level of enzyme troponin in bloodstream that is a direct sign of heart injury. Among these, about one-third was already having CVD. The majority who got covid-19 recovered fully but some people had develop pneumonia and some other diseases. Some patients had "cytokine storm" releasing inflammatory cytokines to blood and damage the multiple organs like heart. ${ }^{1}$
Advanced Covid-19 disease known as "myocarditis". The virus directly invade the heart or may got inflammation due to the release of cytokine. All these causes the enlargement of heart and weakness of heart leads to fluidity in the lungs and low blood pressure. While this severe form of myocarditis is rare, recent studies have suggested that a milder form of heart muscle inflammation may be much more common than previously recognized. Asymptomatic inflammation of heart was observed on magnetic resonance imaging in the patients up to three-quarters after recovery from COVID-19 in a recent study.

\section{Results}

When heart doesn't pump enough blood for body's requirements, then it leads to heart failure. There are a lot of reasons for this happening; heart is too weak to pump properly, heart might can't fill up enough oxygen. "Heart failure" does not mean that it has stopped working. It is a serious condition that needs medical care. ${ }^{2,3}$

A type of lab created stem cells from blood or skin cells used by researchers called human induced pluripotent stem cells (IPSCs).

\begin{tabular}{|l|l|}
\hline Quick Response Code: & *Corresponding author: Sana-e-Mustafa, University of Agriculture, Faisalabad, Pakistan \\
Received: 26 December, 2021 & Published: 30 December, 2021 \\
\hline
\end{tabular}


ISPCs can form any type of cell in the body. Then turned them into heart cells. While exposure to the COVID-19 causing virus, the heart cells were found to be susceptible to infection. It was showed that the virus can divide within the heart muscle cells quickly. Additionally, heart cells changed their gene expression profile, providing new signs that how the body attempts to combat infection. Those people having CVD but adopt healthy behaviors, can make stronger their defenses against COVID-19. They also have reduction for the long-term risk from cardiovascular disease. Intake of a healthy diet along with plenty of physical activity are the key factors. Preventive measures must be obliged as; cooking at home when possible, outdoor walking with friends if gym is temporarily closed, regular measurements of blood pressure at home and continuity to follow the CDC's preventions to wear masks, physical distancing, and avoid large gatherings.

\section{Acknowledgments}

None.

\section{Funding}

None.

\section{Conflicts of interest}

Author declares that there is no conflict of interest.

\section{References}

1. Shi S, Qin M, Shen B, et al. Association of cardiac injury with mortality in hospitalized patients with COVID-19 in Wuhan, China. JAMA Cardiol. 2020

2. Ruan $\mathrm{Q}$, Yang $\mathrm{K}$, Wang $\mathrm{W}$, et al. Clinical predictors of mortal- ity due to COVID-19 based on an analysis of data of 150 patients from Wuhan, China. Intensive Care Med. 2020.

3. Liu Y, Yang Y, Zhang C, et al. Clinical and biochemical indexes from 2019-nCoV infected patients linked to viral loads and lung injury. Sci China Life Sci. 2020;63:364-374. 